\title{
Proposing a Framework Guide for the Integration of Educational Technologies and Innovations into the Teaching of Anatomy and Medical Sciences: The ASIC Framework
}

\section{Joshua Owolabi $\mathbb{D}$}

Department of Anatomy, Division of Basic Medical Sciences, School of Medicine, University of Global Health Equity, Butaro, Burera, Rwanda

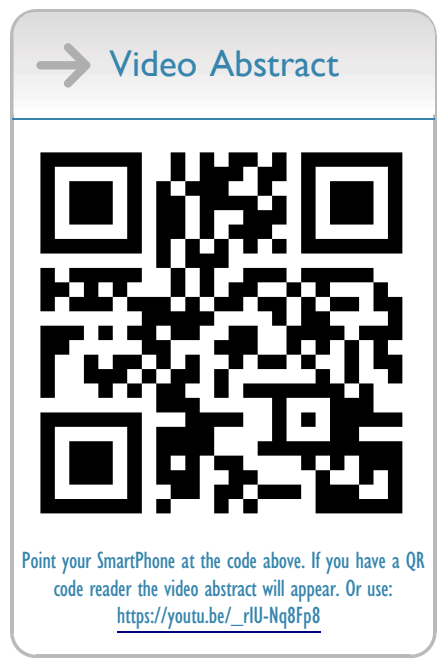

Correspondence: Joshua Owolabi Department of Anatomy, Division of Basic Medical Sciences, School of Medicine, University of Global Health Equity,

Butaro, Burera, Rwanda

Tel +250 78I I64365

Email jowolabi@ughe.org

\begin{abstract}
Educational technologies are becoming important and integral to medical education and practice more than ever before. The scope and appreciation for digital medicine is also widening. No thanks to the covid-19 induced restrictions including physical distancing and lockdown measures, which has changed the mode of educational delivery in many climes. Technology became a major avenue for sustaining medical education and practice. However, what this has also revealed is the heterogeneity in the deployment of technologies and educational innovations based on factors that are linked but not limited to human and system factors. There is currently no generally accepted framework to guide the use of medical education technologies and innovations in the teaching of human anatomy to medical and allied health students. The current framework attempts to provide a framework that could work for various medical education systems, centred on four key considerations: adaptation, standardization, integration, and compliance. In this article, the ASIC framework is presented, illustrated, and succinctly discussed.
\end{abstract}

Keywords: anatomy, education, technology, innovation, adaptation, standardization, integration, compliance

\section{Background}

Educational technologies and innovation have become significant drivers for the delivery of medical education across the world. Interestingly, not much work has been done on the need to provide a generally useful and reliable framework guide for the integration and optimal use of these educational technologies and innovation in the context of medical education. It is also important to note that variations in the types and platforms for educational technologies and innovations have kept increasing. While some have been produced by well-known educational industry players, stakeholders such as faculty members and designers in institutions have equally produced specific educational materials that could be categorized as educational technologies and innovations. These realities when bundled together would point to the fact that there is increasing heterogeneity or variation in the ways and manners that educational innovations and technologies are being adapted and used to deliver medical education across the world. When this is considered in line with the current realities, there has to be a significant level of standardisation, quality assurance and 
best practices that should be achieved in order to globally sustain the quality of medical education delivery. There is a need to provide a framework guide. This article therefore provides the ASIC framework for the adaptation, standardisation, integration of educational technologies and innovations including the need to ensure compliance with best practices within programme structures.

\section{Brief Review of Literature}

Various types of technologies and innovations have been used to teach Anatomy and Basic Medical Sciences, especially in recent times. ${ }^{1-3}$ These had included hardware technologies [eg, the Anatomage Table, the Anatomy Magic Mirror], software [eg, Zygote and the Complete 3D Anatomy software $],{ }^{4}$ virtual realities, ${ }^{5}$ Simulations, ${ }^{6}$ augmented reality facilities [eg, high fidelity mannequin $]^{7,8}$ as well as the use of customised 3D printed materials. ${ }^{9,10}$ These innovations are accompanied by variations in approaches to delivering and facilitating medical education. Remote learning adjusted class structures and blended learning methods have become quite popular. The variations in institutional tech culture, stakeholders embrace versus aversion to technology as well as skills or proficiency levels, availability of supportive systems, leadership, institutional policies, and practices arguably caused significant variations in how technologies and innovations are being used. While it is increasingly becoming clear that technologies and innovations can adequately support and significantly enhance the delivery of medical education, ${ }^{11,12}$ there has been advocacy for regulations and standardisation. The disparities in resources availabilities and the quality of the resources, as well as the systemic support for practical and adequate integration of technologies might be partly due to gaps in funding and infrastructural support between the developed and the developing countries. This has been implicated for why there might have been significant disparities in the deployment of educational technologies and innovations. To be able to set standards, however, there has to be a framework of reference.

\section{The Proposed ASIC Framework}

The ASIC framework, as proposed [Figure 1], provides a guide, developed from extensive consideration for learning theories, pedagogical principles and reflective practice based on experience. It also considers the need to address the heterogeneity in the contexts of deployment of technology educational technologies and innovations. The proposed ASIC framework is based on four key terms Adaptation, Standardisation, Integration and Compliance. This is further illustrated as follows:

A- Adaptation: Innovation and technology should be suitably adapted to programme design and institutional system for optimal performance and best outcomes.

S- Standardisation: Clearly determining the purpose that innovations and technologies serve, the objectives they meet; and supporting their uses with evidence for best and standard practices.

I- Integration: Creating a place for the use of educational innovations and technology; and aligning its use with other components of the educational system for optimal performance.

C- Compliance: Evidence of compliance with institutional standards, programme requirements and regulations of relevant bodies should be addressed.

The ASIC framework [Figure 1] provides guidance on how the four key considerations could be aligned with the cognitive [knowledge], psychomotor [skills] and affective [attitude] aspects of education delivery. Very importantly, it highlights that fact that there should be evidence on how the technology is helping to achieve programme objectives based on outlined milestones. As much as it is practical, each session's objective[s] and the process of accomplishing the objectives should provide an opportunity for the ASIC framework to guide holistic integration of the purpose of use based on the ASIC framework with evidence of such accomplishment in one or more of the KSA domains, as originally premised on programme outcomes and milestones derived from them.

\section{Making a Case for the ASIC Framework}

The implications of negatively disruptive deployment of technology in the teaching of anatomy and medical sciences might be dire and possibly far reaching than anticipated. This proposed framework [Figure 1] could guide medical educators in their use of technology for medical education. Therefore, when deploying educational technologies and innovations, it is therefore important to consider the educational theories, pedagogical principles, and best practices to support their uses for facilitating teaching and training sessions. Finally, with growing interest in educational technologies and innovations as well as digital medicine, developing a tech culture and ensuring proper technology integration have become vital to the 


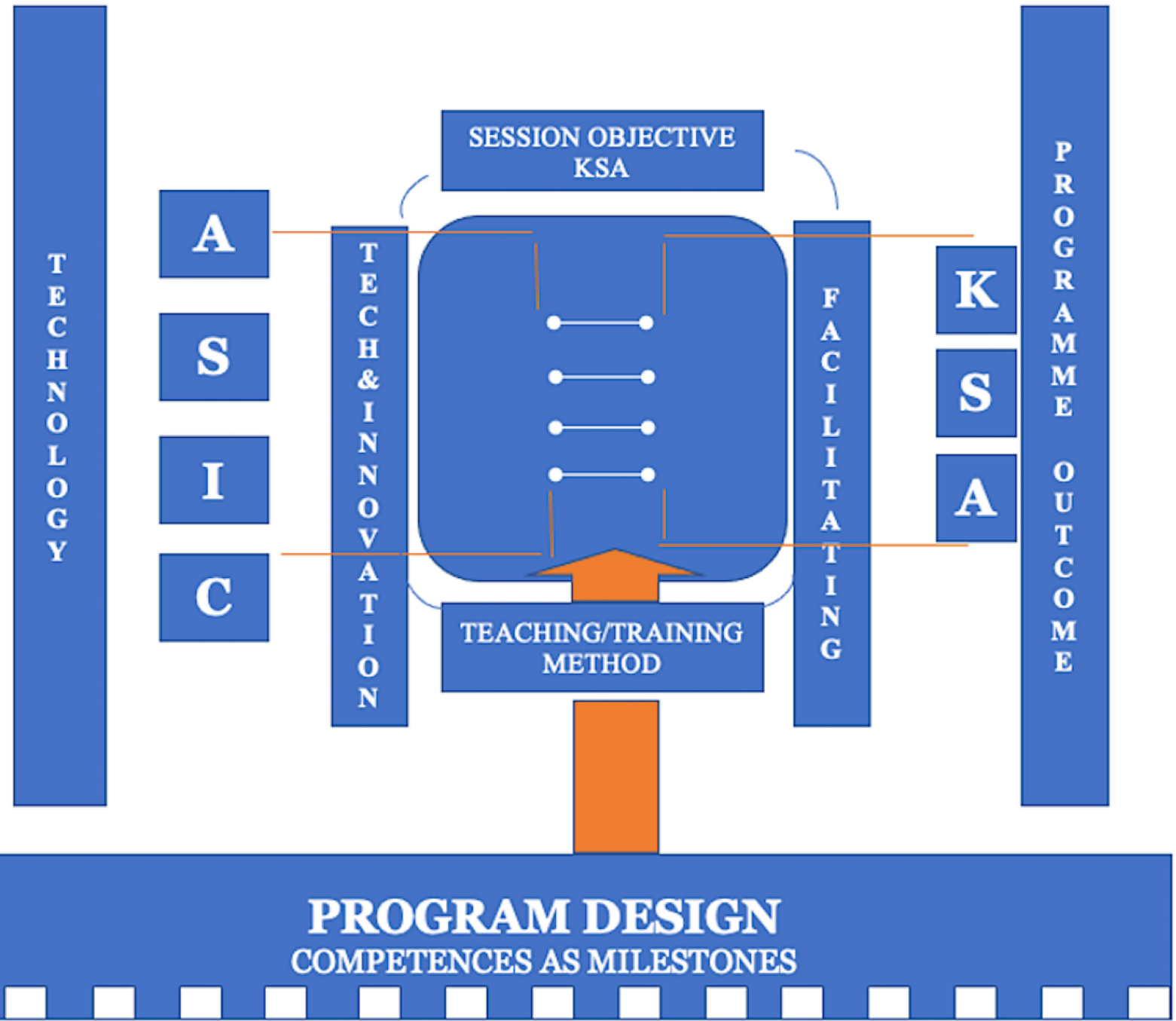

Figure I Figure showing the concept of the ASIC framework. The framework considers four major factors including adaptation [A], standardization [S], integration [I], and compliance [C]. The framework equally provides a guide on how the use of technologies and innovations could be carefully aligned with program outcomes as well as competences and milestones based on program design and expected outcomes.

Abbreviations: K, knowledge; S, skill; A, attitude; ASIC, adaptation [A], standardization [S], integration [I], and compliance [C].

advancement of anatomical sciences and medical education. The following sections further consider the importance of each aspect or tenet of the ASIC Framework.

\section{Adaptation}

The case for adaptation is very important, especially when considering the fact that systems often times vary not just in terms of curricular variant and philosophy, infrastructure and technological setup and support, but also in terms of the culture of learning, policies and standard practices. It would therefore be important to lessen emphasis on uniformity particularly considering the current realities but to ensure proper adaptations that could help to optimise the use of educational technologies and innovations.
When room is provided for adaptation opportunity, adequate latitude will be given to stakeholders to determine how best existing and emerging innovations and technologies can serve the purposes of learning, in line with programme design and objectives. Furthermore, there are variants and sub-variants of medical education curricula which suggest that it might be impractical to expect all systems to use these innovations and technology in exactly the same ways. Therefore, allowing for adaptation would help to suitably deploy educational innovations and technologies in order to achieve specific program objectives as determined by the program stakeholders including the concerned institutions, authorities and regulatory bodies. It is also important to deploy technologies and innovations 
most appropriately and effectively, noting the variations in the purposes that they serve, as well as the strengths and potential deficiencies. $^{13}$

\section{Standardisation}

Standardization will ensure that evidence-based practices are ensured while adopting, adapting, and deploying educational technologies and innovations to deliver medical education. Adhering to the tenets of standardization would emphasize the need to consider appropriate pedagogies while deploying medical education technology. This consideration will also ensure that learning theories are considered. Another way of illustrating the role of standardization is to state that educational technologies and innovation are deployed in practical and scientific manners whereby empirical evidence is used to support practices. For example, the use and choice of augmented reality to teach human anatomy to medical students should be supported by empirical evidence as well as a clear indication of how such a method can help to achieve learning objectives in the context of use. In addition to the need for teachers to ensure the standardisation of their use of educational technologies and innovations, regulatory bodies can also provide a set of guide and requirements for the general use of technologies, yet not limiting creative adaptation and latitude of use. Standardisation is a key consideration in efforts to optimise the benefits of educational technologies and innovations. ${ }^{14}$

\section{Integration}

Integration emphasises the need to use educational technologies and innovations in alignment and in synergy with other technologies and innovations and the entire infrastructure setup to deliver medical education. It is expedient to ensure that the educational technologies and innovations are not negatively disruptive. Proper consideration should be put into how new tools fits into existing systems. Learning tools that are complimentary should specifically meet certain needs with evidence or prospects that their use will enhance the delivery of medical education. Those that are used to replace outdated learning tools and methods including those that are no more feasible and practicable should also satisfy the requirements of their use in specific contexts to deliver medical education. This should be done in line with program objectives whenever possible and necessary. It might be expedient to align their uses with program milestones. Altogether, system thinking should guide the introduction, adaptation and integration of new and emerging educational technologies and innovations into medical education systems. For example, software that have plug-in enablement might be integrated into the learning management system [LMS]. Stand-alone educational technologies and innovation such as the hardware should be allocated specific time in the schedule of events, especially in the syllabus with the specific objectives that their uses will achieve clearly identified and indicated both to students and other stakeholders. The need for adequate integration has been previously highlighted. ${ }^{15}$

\section{Compliance}

Compliance equally emphasizes best practices with specific emphasis on requirements that bother on institutional standards and regulatory bodies requirements, and program outcomes amongst others. For example, certain stand-alone technologies such as the Anatomy magic mirror, the Google Glass and the Anatomage table technology might be great at teaching anatomical concepts, structural organisation, and the required knowledge of functions. Also, the use of high-fidelity manikin might be great at teaching clinical concepts and simulating skills. Therefore, there should be evidence that the use of educational technologies and innovations meet the criteria for compliance as determined by relevant stakeholders and regulatory bodies, especially those with program oversight functions. For the users, the need for compliance would also speak to the need by faculty members and students to have the requisite knowledge and skill to optimally use the tools. ${ }^{16}$ It is in this vein that it might be important that stakeholders and regulatory bodies would expand their regulations to consider use of educational technologies and innovations in order to satisfy the requirement of compliance. This, however, should be done with quality intentions and adequate appreciation for the role and place of educational technologies and innovations, devoid of extreme regimentation that might limit users' creativity and latitude for adaptation.

While there has been quality and increasing evidence about the importance of medical education technologies and innovation in the delivery of medical education and training in the 21 st century, what is equally clear is the need to address specific concerns about how to optimize the use of these technologies and innovations. Standardization is one key term that have stood out. This is not unconnected with the relative heterogeneity in contexts and educational cultures within which these educational technologies and innovations are being deployed. 
Other factors may also be associated with system-related factors such as the quality of infrastructural support, emphasis on educational theories or pedagogical principles, educators' skills in the domain of medical education as well as the quality of medical education leadership amongst other important factors. For example, the need to advance computer assisted instruction through research and roadmap [or framework] has been highlighted. ${ }^{17,18}$

Roadmaps, frameworks, and guiding principles are always important to implementing new educational programmes and strategies. It is, therefore, important to note that the ASIC framework can help to achieve this with regard to medical education technologies and innovations. It can also promote the integration of professionalism into medical education, first with medical educators appreciating the place of learning theories and pedagogical principles in the deployment of medical education technologies and innovation, and consequently imbibing these into the culture of learning and practice of the students and trainees. These could therefore add significant value in the affective domain of learning. It also provides significant value in the domain of the hidden curriculum. Even with traditional practices such cadaveric dissection, professionalism and adherence to ethics that emphasise certain standard practices that are equally supported with quality evidence have been advocated for in the past. ${ }^{19,20}$ Certain hidden curriculum benefits from the use of medical education technologies and innovations following the ASIC Framework would include enshrining a tech-culture in medical education and promoting professionalism with best practices in the use of such technologies and innovations.

\section{Summary and Conclusion}

This framework provides a guide, developed for extensive consideration for learning theories, pedagogical principles and reflective practice based on adequate experience [Figure 1]. It provides a framework for the introduction, effective utilization and optimal deployment of education technologies and innovations to deliver medical education. The framework has also considered four major factors including adaptation, standardization, integration, and compliance. The framework equally provides a guide on how the use of technologies and innovations could be carefully aligned with program outcomes as well as competences and milestones based on program design and expected outcomes. Furthermore, it provides guidance on how the four key considerations could be aligned with the cognitive [knowledge], psychomotor [skills] and affective [attitude] aspects of education delivery. While the framework considers the big picture of the entire medical education delivery, it also considers the need to give adequate considerations to these factors with every opportunity to use educational technologies and innovations such as in classrooms as well as simulation rooms. It should be stated, finally, that while this framework is recommended for use, continuous effort is being invested into further studies to validate and improve the effectiveness of its use as a reference guide.

\section{Disclosure}

The author reports no conflicts of interest in this work.

\section{References}

1. Fallavollita P. Innovative technologies for medical education, human anatomy - reviews and medical advances, Alina Maria Sisu. IntechOpen. 2017. doi:10.5772/intechopen.68775

2. Zargaran A, Turki MA, Bhaskar J, Spiers H, Zargaran D. The role of technology in anatomy teaching: striking the right balance. Advan Med Educ Pract. 2020;11:259-266. doi:10.2147/AMEP.S240150

3. Dawidziuk A, Kawka M, Szyszka B, Wadunde I, Ghimire A. Global access to technology-enhanced medical education during the COVID-19 pandemic: the role of students in narrowing the gap. Glob Health Sci Pract. 2021;9(1):10-14. doi:10.9745/GHSP-D-2000455

4. Santana EA, Orquera PA, Valenzuela JJ, Orellana MI, Gold MH, De La Paz Garcia G. Anatomical software as a tool in the teaching-learning process of human anatomy. Literature review. FASEB J. 2020;34:1. doi:10.1096/fasebj.2020.34.s1.09262

5. Zhao J, Xu X, Jiang $\mathrm{H}$, et al. The effectiveness of virtual reality-based technology on anatomy teaching: a meta-analysis of randomized controlled studies. BMC Med Educ. 2020;20:127. doi:10.1186/s12909-020-1994-Z

6. Kurt E, Yurdakul SE, Ataç A. An overview of the technologies used for anatomy education in terms of medical history. Procedia. 2013;103:109-115. doi:10.1016/j.sbspro.2013.10.314

7. Campisi CA, Li EH, Jimenez DE, Milanaik RL. Augmented reality in medical education and training: from physicians to patients. In: Geroimenko V, editor. Augmented Reality in Education. Springer Series on Cultural Computing. Cham: Springer; 2020. doi:10.1007/ 978-3-030-42156-4_7.

8. Parsons D, MacCallum K. Current perspectives on augmented reality in medical education: applications, affordances and limitations. $A d v$ Med Educ Pract. 2021;12:77-91. doi:10.2147/AMEP.S249891

9. McMenamin PG, Quayle MR, McHenry CR, et al. The production of anatomical teaching resources using three-dimensional (3D) printing technology. Anat Sci Educ. 2014;7(6):479-486. doi:10.1002/ase.v7.6

10. Reyes GJP, Valle TCJ, Soto UV, Herrera VI, Pineda MD, Garcia GAA. New technologies applied to the study and application of digital anatomy. HDM project (human dissection models)- 5 years of experience. Ann Med. 2019;51:sup1,56. doi:10.1080/ 07853890.2018 .1561926

11. Goh PS. eLearning or technology enhanced learning in medical education - hope, not hype. Med Teacher. 2016;38(9):957-958. doi:10.3109/0142159X.2016.1147538

12. Owolabi J, Bekele A. Implementation of innovative educational technologies in teaching of anatomy and basic medical sciences during the COVID-19 pandemic in a developing country: the COVID-19 silver lining? Adv Med Educ Pract. 2021;12:619-625. doi:10.2147/AMEP.S295239 
13. Guze PA. Using technology to meet the challenges of medical education. Trans Am Clin Climatol Assoc. 2015;126:260-270.

14. Moran J, Briscoe G, Peglow S. Current technology in advancing medical education: perspectives for learning and providing care. Acad Psychiatry. 2018;42:796-799. doi:10.1007/s40596-018-0946-y

15. Han ER, Yeo S, Kim MJ, et al. Medical education trends for future physicians in the era of advanced technology and artificial intelligence: an integrative review. BMC Med Educ. 2019;19:460. doi:10.1186/s12909-019-1891-5

16. Grimwood T, Snell L. The use of technology in healthcare education: a literature review'. MedEdPublish. 2020;9(1):137. doi:10.15694/ mep.2020.000137.1

17. Berman NB, Fall LH, Maloney CG, et al. Computer-assisted instruction in clinical education: a roadmap to increasing CAI implementation. Adv Health Sci Educ. 2008;13:373-383. doi:10.1007/s10459-006-9041-3
18. Cook DA. The research we still are not doing: an agenda for the study of computer-based learning. Acad Med. 2005;80(6):541-548. doi:10.1097/00001888-200506000-00005

19. Ghosh SK. The practice of ethics in the context of human dissection: setting standards for future physicians. Ann Anat. 2020;232:151577. doi:10.1016/j.aanat.2020.151577

20. Ghosh SK, Kumar A. Building professionalism in human dissection room as a component of hidden curriculum delivery: a systematic review of good practices. Anat Sci Educ. 2019;12(2):210-221. doi:10.1002/ase.1836

\section{Publish your work in this journal}

Advances in Medical Education and Practice is an international, peerreviewed, open access journal that aims to present and publish research on Medical Education covering medical, dental, nursing and allied health care professional education. The journal covers undergraduate education, postgraduate training and continuing medical education including emerging trends and innovative models linking education, research, and health care services. The manuscript management system is completely online and includes a very quick and fair peer-review system. Visit http://www.dovepress.com/testimonials.php to read real quotes from published authors. 